\title{
Microwave absorbing properties of activated carbon fibre polymer composites
}

\author{
TIANCHUN ZOU*, NAIQIN ZHAO ${ }^{\dagger}$, CHUNSHENG SHI' and JIAJUN LI ${ }^{\dagger}$ \\ Airworthiness Certification Technology Research and Management Centre, Civil Aviation University of China, \\ Tianjin 300300, P.R. China \\ ${ }^{\dagger}$ School of Materials Science and Engineering, Tianjin University, Tianjin 300072, P.R. China
}

MS received 10 August 2009; revised 22 January 2010

\begin{abstract}
Microwave absorption of composites containing activated carbon fibres (ACFs) was investigated. The results show that the absorptivity greatly depends on increasing ACF content in the absorbing layer, first increasing and then decreasing. When the content is $0.76 \mathrm{wt} \%$, the bandwidth below $-10 \mathrm{~dB}$ is $12.2 \mathrm{GHz}$. Comparing the absorption characteristics of the ACF composite with one containing unactivated fibres, it is found that carbon fibre activation increases the absorption of the composite.
\end{abstract}

Keywords. Activated carbon fibres; microwave absorbing properties; composite materials.

\section{Introduction}

The reduction of electromagnetic backscatter with the use of microwave absorbing material (MAM) has important applications in the field of radar and electromagnetic compatibility (Singh et al 2006; Qiu et al 2007). Carbon materials, which are considered as the most promising absorbent since World War II, has been widely employed in microwave absorbing materials in reducing backscattering from objects or radar targets, EMI suppressors and paints (Xie et al 2005; Zhang and Zhu 2009). More recently, it has been reported that activation of carbon fibres can enhance the contribution of multiple reflections and increase the EMI shielding effectiveness for carbon fibre composites ( $\mathrm{Wu}$ and Chung 2002). However, so far, little has been reported on the electromagnetic wave absorbing properties of activated carbon fibres (ACFs) in the open literature. In this work, discontinuous activated carbon fibres (ACFs) are used as the absorbent. By changing $\mathrm{ACF}$ content in the absorbing layer, different composites are prepared. The experimental evaluation of composites is presented, and the influence of ACF content on absorption is analysed. To investigate the effect of activation on the reflection properties, the absorbing performances of the composite containing ACFs and that with unactivated ones are also compared.

\section{Experimental}

\subsection{Materials}

The epoxy resin (E-44, Resin company in Wuxi, PRC) cured with the polyamide resin $\left(203^{\#}\right.$, Chemical plant in Tianjin, PRC) was adopted as the matrix of the samples. The epoxy resin has a permittivity of $3 \cdot 0-3 \cdot 4$ and the dielectric loss tangent, $0 \cdot 01-0 \cdot 03$. The polyamide resin has a permittivity of 3.0-4.0 and electrical resistivity of $10^{11}-10^{12} \Omega \cdot \mathrm{m}$. Viscose-based ACFs with average length of $\sim 32 \mathrm{~mm}$, average diameter of 13-16 $\mu \mathrm{m}$ and specific surface area of $823 \mathrm{~m}^{2} \mathrm{~g}^{-1}$ was used as the absorbent in composites. The fibres have irregular-shaped cross sections (shown in figure 1), which are very advantageous for the absorption of electromagnetic wave (Zhao et al 2001).

\subsection{Preparation of ACFs}

Viscose fibres (the precursors) carbonization and activation were conducted by (i) pre-soaking the precursors in an aqueous solution $\left[\mathrm{NH}_{4} \mathrm{H}_{2} \mathrm{PO}_{4} 3 \mathrm{wt} \%\right.$, $\left.\left(\mathrm{NH}_{4}\right)_{2} \mathrm{SO}_{4} 15 \mathrm{wt} . \%\right]$ for the purpose of surface cleansing and improving carbon yields of activated materials, (ii) heating the pre-soaked fibres in nitrogen gas $(99.99 \%, 133-266 \mathrm{~Pa})$ to $450^{\circ} \mathrm{C}$ at a heating rate of $20^{\circ} \mathrm{C} / \mathrm{min}$, (iii) maintaining the temperature at $450^{\circ} \mathrm{C}$ for $30 \mathrm{~min}$ for the purpose of carbonization, (iv) heating the fibres to $950^{\circ} \mathrm{C}$ at a speed of $50^{\circ} \mathrm{C} / \mathrm{min}$, (v) introducing steam (with a partial pressure of $20 \%$ ) for 10 min while the temperature remained at $950^{\circ} \mathrm{C}$ for the purpose of activation, and (vi) cooling to room temperature. In this paper, unactivated

\footnotetext{
*Author for correspondence (zoutianchun@yahoo.com.cn)
} 
carbon fibres are the resulting fibres before step (iv), i.e. viscose fibres were only carbonized, not activated.

\subsection{Preparation of composites}

ACF composites, consisting of the absorbing and transparent layers, were fabricated by the die-pressing process. ACFs were blended uniformly with the epoxy resin in a definite ratio. Then the polyamide resin was poured into the ACF paste in the mass ratio of 1:2 while stirring. After vacuum deformation the mixture was cast into a semi-overflowing 1045 steel die layer-by-layer until its thickness reached $3 \mathrm{~mm}$, which would form the absorbing layer of the sample. Another blend consisting of the epoxy and polyamide resins in the mass ratio of 2:1 was evenly coated on the absorbing layer until total thickness of the sample reached $4 \mathrm{~mm}$. The layer thickness of $1 \mathrm{~mm}$, consisting of the polyamide and epoxy resins, became the transparent layer. Molding was carried out in a hydraulic press at $10 \mathrm{MPa}$ pressure and $80^{\circ} \mathrm{C}$ for $2 \mathrm{~h}$, obtaining specimens of $500 \times 500 \times 4 \mathrm{~mm}$ for reflectivity measurement. The configuration of the sample is shown in figure 2.

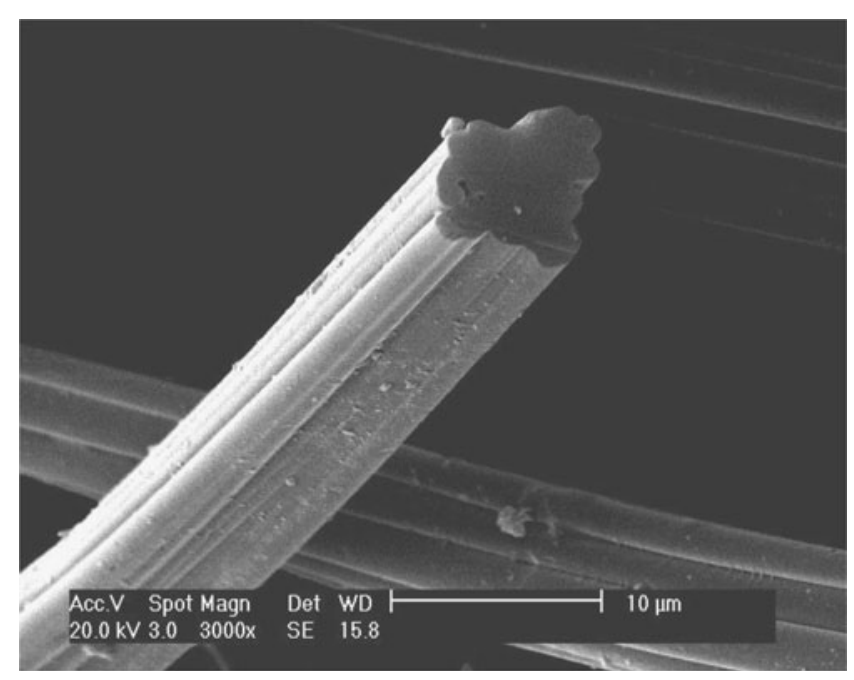

Figure 1. SEM photograph of viscose-based ACFs.

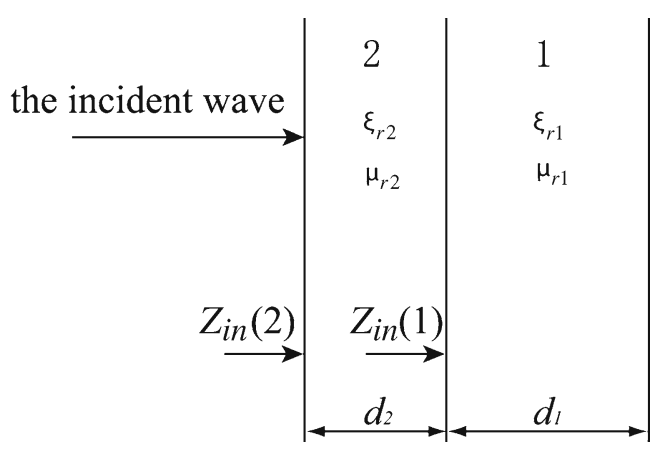

Figure 2. Cross-section of the sample (1. the absorbing layer; 2. the transparent layer).
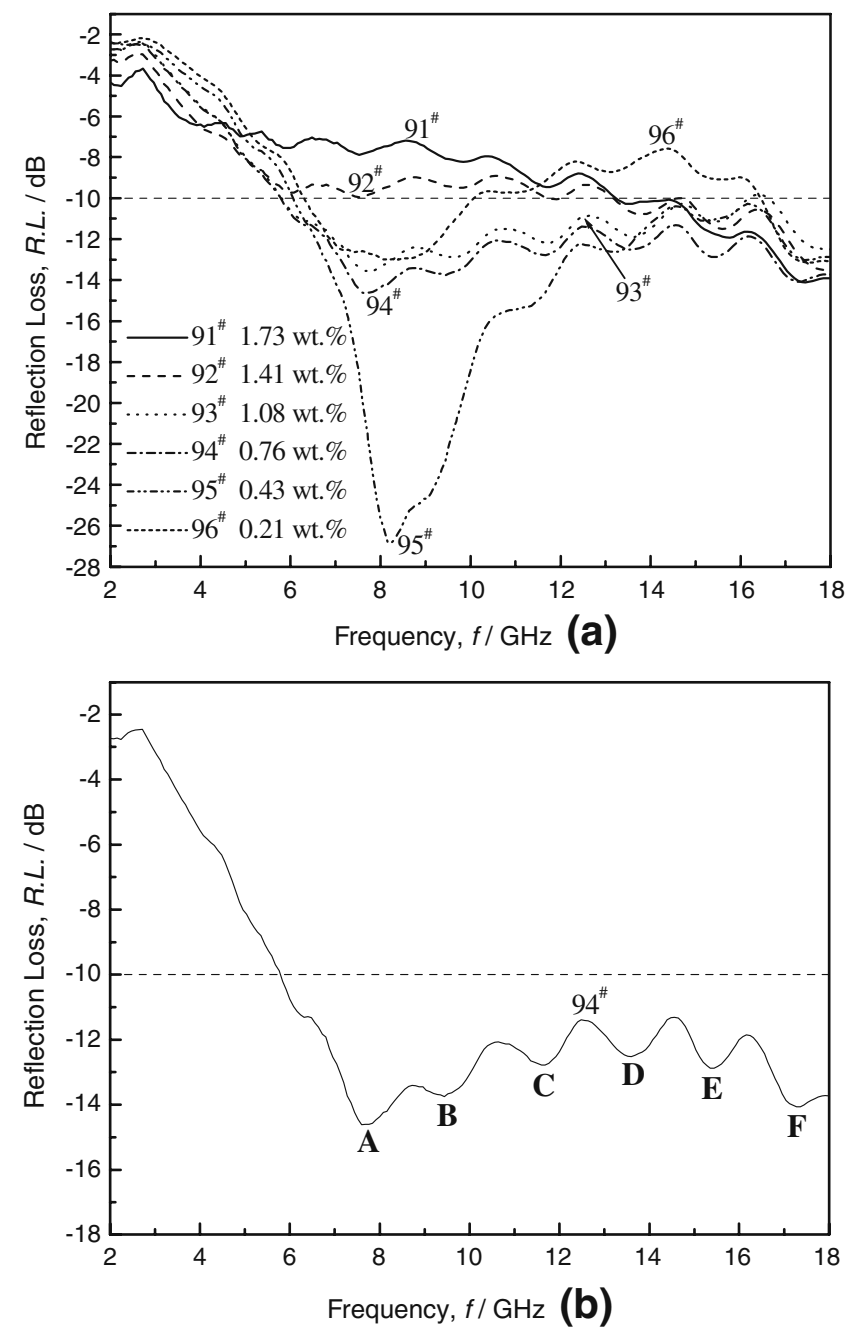

Figure 3. Effect of ACF content on RL.

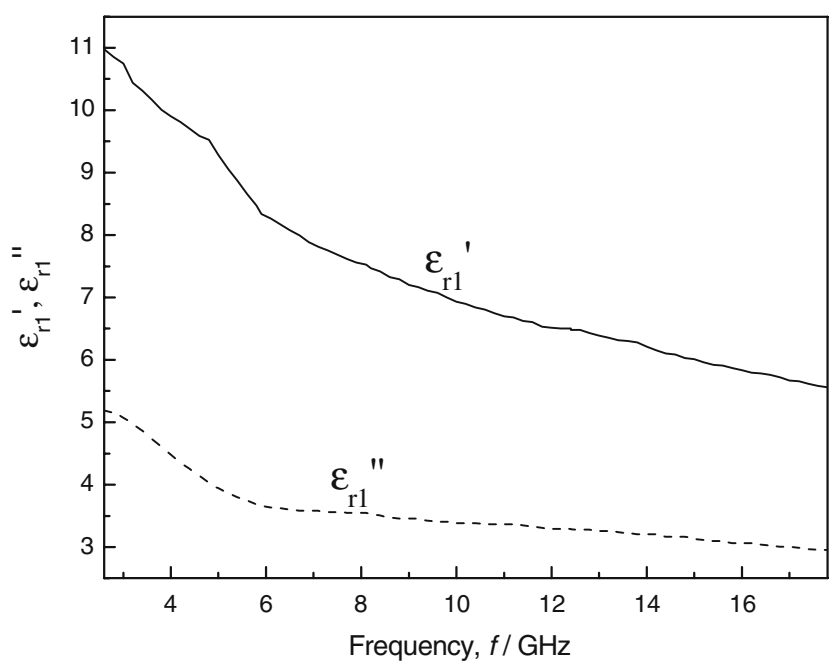

Figure 4. Real $\varepsilon_{r}^{\prime}$ and imaginary $\varepsilon_{r}^{\prime \prime}$ parts of the permittivity of the absorbing layer for specimen $95^{\#}$. 


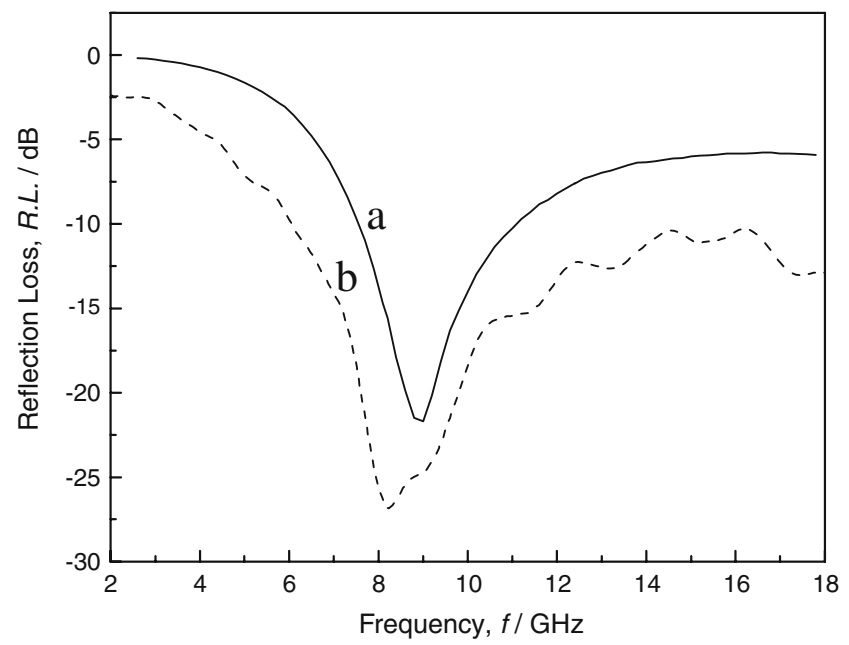

Figure 5. Comparison between the calculated and measured RL for specimen $95^{\#}$ (a. calculated values and b. measured values).

\subsection{Measurements of microwave absorption}

The absorption characteristics of the samples were measured over the frequency range $2-18 \mathrm{GHz}$ in a calibrated NRL (Naval Research Laboratory) arch using a HP8757E network analyser (Knott et al 1985). The sample under test was positioned on a aluminum panel $(500 \times 500 \mathrm{~mm})$. The transmission/reflection $(T / R)$ rectangular waveguide method was employed to determine the microwave permittivity in the frequency range $2 \cdot 6-17.8 \mathrm{GHz}$ (Wu et al 2000).

\section{Results and discussion}

\subsection{Effect of ACF content on reflection loss $(R L)$ of composites}

Figure 3 shows the frequency dependence of RL of composites containing different weight percentages of ACFs in the absorbing layer. It can be seen that ACF content influences the RL of composites greatly. As shown in figure 3, the minimum RL decreases and the bandwidth below $-10 \mathrm{~dB}$ increases while ACF content decreases from $1.73 \mathrm{wt} . \%-0.76$ wt. $\%$. As the content is 0.76 wt. $\%$ (sample $94^{\#}$ ), the RL fluctuates around $-12.5 \mathrm{~dB}$ in the frequency range from 5.8 $18 \mathrm{GHz}$. After that, with the content continuing to decrease, the absorbing effect of composites begins to fall. Obviously, the optimum RL corresponded to a suitable ACF content, which is around $0.76 \mathrm{wt} \%$ in this case. It can also be seen that in the frequency region $7 \cdot 5-18 \mathrm{GHz}$, the absorp-

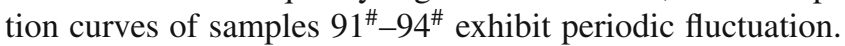
For example, the absorption curve of sample $94^{\#}$ has six small hollows marked with $\mathrm{A}-\mathrm{F}$ in this frequency region (see figure 3 (b)). The frequencies corresponding to the minimum RL values of hollows A-E are 7.60 GHz, 9.44 GHz, 11.68 GHz, $13.6 \mathrm{GHz}, 15.36 \mathrm{GHz}$ and $17.28 \mathrm{GHz}$. The difference between adjacent frequencies is approximately identical (A-B $1.84 \mathrm{GHz}, \mathrm{B}-\mathrm{C} 2.24 \mathrm{GHz}, \mathrm{C}-\mathrm{D} 1.92 \mathrm{GHz}, \mathrm{D}-\mathrm{E}$ $1.76 \mathrm{GHz}, \mathrm{E}-\mathrm{F} 1.92 \mathrm{GHz}$ ) and the average value is $1.94 \mathrm{GHz}$. The frequencies corresponding to the minimum RL values

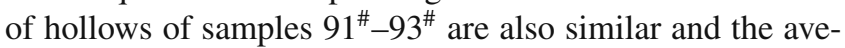
rage difference between adjacent frequencies are $1.98 \mathrm{GHz}$, $2.13 \mathrm{GHz}$ and $2.08 \mathrm{GHz}$, respectively.

Take samples $94^{\#}$ and $95^{\#}$ for examples to discuss the microwave absorbing mechanism of ACF composites. According to the transmission-line theory (Meshram et al 2003), the RL is related to the input impedance, $Z_{\text {in }}(2)$, of a metal-backed two-layer microwave absorbing materials (see figure 2) as

$$
\mathrm{RL}=20 \lg \left|\frac{Z_{\text {in }}(2)-Z_{\mathrm{C}}^{0}}{Z_{\text {in }}(2)+Z_{\mathrm{C}}^{0}}\right|,
$$

where $Z_{\mathrm{C}}^{0}$ is the wave impedance of free space $(376 \cdot 7 \Omega), Z_{\text {in }}$ (2) is given by

$$
Z_{\text {in }}(2)=Z_{\mathrm{C}}^{m}(2) \frac{Z_{\text {in }}(1)+Z_{\mathrm{C}}^{m}(2) \operatorname{th} \gamma(2) d_{2}}{Z_{\mathrm{C}}^{m}(2)+Z_{\text {in }}(1) \operatorname{th} \gamma(2) d_{2}},
$$

where the input impedance of the first layer, $Z_{\text {in }}(1)=$ $Z_{\mathrm{C}}^{m}$ (1) $t h \gamma(1) d_{1}$, the intrinsic impedance of the $k$ th layer, $Z_{\mathrm{C}}^{m}(k)=\sqrt{\frac{\mu_{0}}{\varepsilon_{0}}} \sqrt{\frac{\mu_{r k}}{\varepsilon_{r k}}}(k=1,2)$, the propagation constants of the $k$ th layer, $\gamma(k)=j \frac{2 \pi f}{c} \sqrt{\mu_{r k} \varepsilon_{r k}}(c$ is the light velocity in free space), $\mu_{0}$ and $\varepsilon_{0}$ are the permeability and permittivity of free space, $\mu_{r k}, \varepsilon_{r k}$ and $d_{k}$ are the relative complex permeability, permittivity and thickness of the $k$ th layer, respectively. In the frequency region $2.6-17.8 \mathrm{GHz}, \mu_{r 2}=1.0$, $\varepsilon_{r 2}=2 \cdot 8, \mu_{r 1}=1 \cdot 0$ and the complex permittivity $\left(\varepsilon_{r 1}^{\prime}, \varepsilon_{r 1}^{\prime \prime}\right)$ of the absorbing layer is shown in figure 4 .

The theoretical RL was calculated by a computer simulation using (1) and (2) based on the measured values of $\varepsilon_{r}$ and $\mu_{r}$. For comparison, the calculated and measured RL curves are illustrated in figure 5. As can be seen from figure 5, the shapes of experimental and calculated curves are similar and there is only a small difference in $\mathrm{RL}(\sim 5 \mathrm{~dB})$ between them, which indicates that dielectric loss is the main mechanism

Table 1. Dielectric properties of absorbing layer for sample $95^{\#}$.

\begin{tabular}{lcccccr}
\hline$f / \mathrm{GHz}$ & 7.60 & 9.44 & 11.68 & 13.6 & $15 \cdot 36$ & $17 \cdot 28$ \\
\hline$\varepsilon_{r 1}^{\prime}-j \varepsilon_{r 1}^{\prime \prime}$ & $93.63-j 5.11$ & $59.27-j 4.86$ & $38.95-j 4.53$ & $27.25-j 4.29$ & $23.67-j 3.89$ & $18 \cdot 12-j 3.53$ \\
\hline
\end{tabular}


Table 2. Comparison between $f$ corresponding to minimum RL in hollows A-E and $f$ corresponding to interference counteraction.

\begin{tabular}{|c|c|c|c|c|c|c|}
\hline Hollows & A & $\mathrm{B}$ & $\mathrm{C}$ & $\mathrm{D}$ & $\mathrm{E}$ & $\mathrm{F}$ \\
\hline Corresponding to minimum RL in hollows $(f / \mathrm{GHz})$ & $7 \cdot 60$ & $9 \cdot 44$ & $11 \cdot 68$ & $13 \cdot 6$ & $15 \cdot 36$ & $17 \cdot 28$ \\
\hline Corresponding to interference counteraction $(f / \mathrm{GHz})$ & $7 \cdot 19$ & $9 \cdot 66$ & $12 \cdot 10$ & $13 \cdot 58$ & $15 \cdot 62$ & 17.59 \\
\hline
\end{tabular}

for absorption of microwave. However, the theoretical curve does not fit exactly on the measured curve. The discrepancy may be attributed to: (i) instead of the apparent interface, a thin transition layer forms between the transparent and absorbing layers by employing integrally manufacturing technique in sample preparation, which makes electromagnetic parameters exhibit a smooth and continuous change at the two layers' interface. This leads to improvement of the microwave absorbing properties and the shift of the absorption band towards low frequencies, and (ii) the length of ACFs $(\sim 32 \mathrm{~mm})$ used in this work is close to the wavelength of incident wave (16.6-150 mm). When incident wave arrives at ACFs, high-frequency currents are excited in the fibres and electromagnetic energy is partly transformed into heat energy by the resistance loss.

The complex permittivity of the absorbing layer of sample $95^{\#}$ at the frequency corresponding to the minimum RL value of hollows A-E (see figure 3 (b)) is presented in table 1. The absorbing layer shows much greater permittivity in comparison to the transparent layer, so when considering interference counteraction, the effect of the transparent layer can be neglected. As the incident and reflected waves in the absorbing layer are out of phase by $180^{\circ}$, the reflected wave is partly attenuated at the surface of the absorbing layer and the matching frequency is given by the following expression (Yusoff and Abdullah 2004):

$$
f=\frac{2 m+1}{4 d \sqrt{\left|\varepsilon_{r 1}\right|}} c \quad m=0,1,2 \ldots \ldots \ldots,
$$

where the thickness of the absorbing layer, $d=3 \mathrm{~mm}$, and $\left|\varepsilon_{r 1}\right|$ is the modulus of $\varepsilon_{r 1}$. The frequency values corresponding to interference counteraction are calculated using (3) from the parameters listed in table 1. The comparison between calculated values and those corresponding to the minimum RL of hollows A-E is shown in table 2 . It is found that two groups of frequencies are very close, which indicates that interference counteraction is the reason for periodic fluctuation of absorption curves in higher frequencies.

From the experimental results, it is suggested that ACF content in composites has the optimum value for microwave absorption. This is because when ACF content is very low, dielectric loss is too small to consume electromagnetic energy effectively. However, with the increase of ACF content, composites gradually present strong reflection characteristics, resulting in a decrease of the absorptivity.

\subsection{Effect of activation on RL of composites}

In order to evaluate the effect of activation on the absorption, the composite with $0.43 \mathrm{wt} . \%$ unactivated carbon fibres in the absorbing layer (sample $97^{\#}$ ) was also prepared. Figure 6 shows comparison between RL of sample $95^{\#}$ and that of the one containing unactivated fibres. It can be observed that compared with the latter, the composite containing ACFs has better absorbing properties, while the content of fibres keeps unchangeable. The maximum absorption of the composite with unactivated fibres is $-10.2 \mathrm{~dB}$ at $11.9 \mathrm{GHz}$. But the maximum absorption of sample $95^{\#}$ reaches $-26.8 \mathrm{~dB}$ at $8 \cdot 2 \mathrm{GHz}$ and the bandwidth below $-10 \mathrm{~dB}$ of about $12 \mathrm{GHz}$ is obtained. After activation the contribution by multiple reflections is improved (Wu and Chung 2002). This means that the electromagnetic wave has a longer propagating distance in composites and is more attenuated.

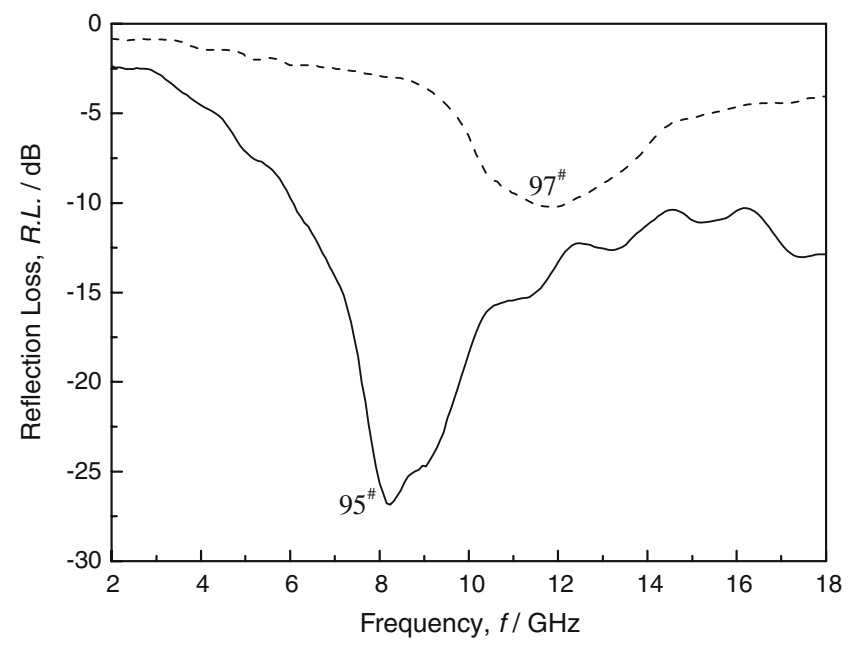

Figure 6. Effect of carbon fibre activation on RL of composites. 


\section{Conclusions}

ACFs would be a kind of promising absorbent for microwave absorption. Composites containing ACFs exhibit good absorption in the frequency range 5-18 GHz. The absorption is strongly dependent on the ACF content. With optimum loading the composite shows a reflection loss below $-10 \mathrm{~dB}$ in the frequency region 5.8-18 GHz. Compared with unactivated carbon fibres, composites containing ACFs show better absorbing properties, which indicates that activating carbon fibres is an effective method to improve the absorbing effectiveness.

\section{Acknowledgement}

The work is supported by Doctor Fund from the Educational Ministry of China (No. 20050056041).

\section{References}

Knott E F, Schaeffer J F and Tuley M T 1985 Radar cross section (New Jersey: Artech House Inc.)

Meshram M R, Agrawal N K, Sinha B and Misra P S 2003 J. Magn. Magn. Mater. 271207

Qiu J X, Wang Y and Gu M Y 2007 J. Mater. Sci. 42166

Singh P, Babbar V K, Razdan A, Srivastava S L, Agrawal V K and Goel T C 2006 J. Mater. Sci. 417190

Wu J H and Chung D D L 2002 Carbon 40445

Wu M Z, Zhao Z S, He H H and Yao X 2000 J. Magn. Magn. Mater. 21789

Xie G W, Wang Z B, Cui Z L and Shi Y L 2005 Carbon 43 3181

Yusoff A N and Abdullah M H 2004 J. Magn. Magn. Mater. 269 271

Zhang L and Zhu H 2009 Mater. Lett. 63272

Zhao D L, Shen Z M and Chi W D 2001 New Carbon Mater. 16 66 